\title{
NEUROSES AND PSYCHONEUROSES.
}

[163] Encephalitic and hysterical syndromes (Sindromi encefalitiche e sindromi: isteriche patogenesi).--V. M. Buscaino. Riv. di pat. nerv. e ment., 1932, xxxix, 33.

THE author has studied the literature of non-purulent encephalitis especially in relation to oculogyric crises and drawn from it the evidence which can best contribute to the study of the genesis of the hysterical syndromes. He comes to the general conclusion that the extracortical zone (basal ganglia and mesencephalon) is of major importance in the genesis of the most important elements in the hysterical syndrome.

These conclusions are at present general and must be worked out in greater detail later, with the documentation of the objective disturbances of the vegetative innervation of the hysteric-disturbances quite independent of psychic activity (especially in relation to the asymmetry of vegetative innervation between the two sides of the body). Attention must also be given to the documentation of hysterical disturbances of an emotional and vegetato-emotional character with special relation to the common site of emotional and vegetative activity in the basal ganglia and mesencephalon.

R. G. G.

\section{PSYCHOSES.}

[164] A note on the relationship between the blood cholesterol and hyperglycæmic index in manic-depressive psychosis.-MADELINE $R$. Lockwood. Jour. of Ment. Sci., 1932, lxxviii, 901.

THE hyperglycæmic index and the blood cholesterol were examined in 24 cases of manic-depressive psychosis, and a relationship was shown to exist between the two values. The blood cholesterol content shows a delay in the return to normal as compared with the glucose tolerance curve. When the hyperglycæmic index falls to zero, the cholesterol level lies at the upper limit of normal. A rise in the index is associated with quantitative changes in the blood cholesterol of such a nature that the two values bear an inverse ratio to each other. Three cases in which the test has been repeated at different phases of the illness show that variations in the index are accompanied by variations in the cholesterol level. The possible relationship between these findings and a disordered endocrine balance is briefly discussed and an explanation suggested.

C. S. R.

[165] Psychosis : its importance as a presenting symptom of brain tumour.Leo J. Adelstein and Martin G. Carter. Amer. Jour. Psychiat., 1932, xii, 317.

THE incidence of brain tumour as a clinical entity is approximately 1 per cent. Psychosis per se may be the first presenting symptom. All cases 
presenting a psychosis should receive a thorough neurological examination to rule out a possible organic basis in the nature of an intracranial neoplasm. Visual and auditory hallucinations are of localizing value only if distinct and apart from a psychosis. Mental phenomena associated with brain tumour may be regarded as focal in nature only if the neurological examination will bear out the localization. In the series of cases here studied, the most marked mental changes were noted in those involving the frontal lobes and the corpus callosum, which coincides with the opinion of various authorities.

C. S. R.

[166] A study of the sexual life in psychoses associated with childbirth.E. W. Anderson. Jour. of Ment. Sci., 1933, lxxix, 137.

FifTy cases of psychosis, occurring in association with childbirth, were studied with special reference to the sexual life, and compared with a like number of cases of psychosis in married women of approximately the same age-period where the puerperium was not the precipitating factor. No material differences were revealed in the prepsychotic sexual life of the two groups. Persistent frigidity cannot be reckoned a distinctive or even constant feature of the sexual constitution of the cases in the puerperal group. Masturbation and eroticism were only slightly commoner in the puerperal group, and cannot be regarded as characteristic or even particularly common features of the group. In the whole series of 100 cases masturbation only occurred in eight. The attitude to both husband and child in puerperal psychoses is inconstant, and aversion to either or both is by no means constantly or even very frequently found. The figures relating to previous attacks taken in conjunction with those on the prepsychotic sexual life, fail to support the view that the onset of a psychosis associated with childbirth is a product of a morbid sexual constitution, with the puerperium acting as a specific precipitating factor. The latter must be regarded in the same way as any other precipitating factor whatever.

C. S. R.

[167] Use of carbon dioxide mixtures in stupors occurring in psychoses.F. C. D'Elseaux and H. C. Solomon. Arch. of Neurol. and Psychiat., 1933, xxix, 213.

The essential purpose of the authors has been to determine the cause or causes instrumental in arousing patients from their stuporous condition when they are given carbon dioxide to inhale. This arousal has been called the mental response, and on the evaluation of it depend the judgment as to the relative efficacy of the various procedures used and the judgment as to what are the factors causative in bringing it about.

One is forced to rely on the overt behaviour of the patient for making this estimation. This includes muscular movement of both the voluntary 
and the involuntary sort, verbal utterances and such elusive yet real factors as the gleam in the eye and modulation of the voice. Furthermore, there are a number of attributes of each of these forms of activity which are used in estimating the mental activity of a person. Some are the degree of spontaneity of the act, or, on the other hand, the amount of stimulation needed to produce it; the relative complexity of the movement or verbal utterance and of the stimulus calling it out; the speed of initiation and of performance, and the completeness of performance; the purposiveness, adequacy and degree of relevancy of the act to the stimulus and to the total situation.

Besides the degree of normality, two other factors must be considered: (1) the amount of response obtained or, in other words, the increase in overt behaviour over that present in rest, and (2) the duration of the response, that is, the length of time during which the change in overt behaviour is present.

In addition to carbon dioxide, experiments were made with nitrous oxide and diathermy. The stimulating effect on catatonic patients of all these different methods indicated that no one or no combination of the observed physiological changes can be considered as essential to the obtaining of the response; the multiplicity of methods used with success hardly admits of a single and common physiological factor being responsible; the only known similarity between these procedures which may be responsible for the effect is some sort of shock to the body economy; yet other such shocks are not effective, nor are these always effective. There are various psychological factors which alter the response, at times increasing it or insuring it and at other times decreasing or even negating it. The various a priori hypotheses that have been advanced to explain the effect obtained, such as stimulation of the nerve structures by carbon dioxide or by a difference in the supply or utilization of oxygen, or a change in the metabolic and functional activity of the nerve cells as a result of changes in acidity, lack factual evidence for their support; they are neither proved nor disproved, but it can be said definitely that none of them is adequate to explain the phenomena in question.

R. M. S.

\section{[168] A clinical study of psychoses associated with various types of endo- crinopathy._J. Norkin. Amer. Jour. Psychiat., 1932, xii, 331.}

THis study indicates that the occurrence of psychoses in association with endocrinopathies of gross character is rather infrequent, inasmuch as only eight cases were found in a hospital population of 6,000 patients. The presence of three hypophrenics in this group reduces this number to a greater extent, as the endocrinopathies in mental deficiency are not infrequent. This infrequency seems to indicate that the relationship of the 
endocrine dysfunction to mental disorders has been overrated considerably, especially if we bear in mind the frequent occurrence of endocrine dysfunction without psychoses. The stress which has been put upon certain disturbances in calcium and lipoid metabolism (cholesterol, lecithin) and offered as a possible etological factor in mental disorders does not seem to be justified, as the deviations for calcium and lipins in such overt endocrinopathies as are presented in this study are minimal. The lack of any definite findings on which an organic etiology can be built up does not entail having recourse to psychoanalytic doctrine. It seems, however, reasonable to assume that in a number of these cases the physical handicap is tied up with the dynamics of the mental trend.

C. S. R.

\section{PSYCHOPATHOLOGY.}

[169] Crime and the endocrine glands.-Louis Berman. Amer. Jour. Psychiat., 1932, xii, 215.

This writer regards crime as due, in a ' Gestalt' sense, to a perversion of the instinctive drives dependent upon a deficiency and imbalance of the endocrine glands. Certain types of crimes are associated with certain types of endocrine malfunctioning. Most criminals are derived from juvenile delinquents and most juvenile delinquents tend to become criminals. Endocrine imbalance and deficiency have been found to occur in about the same frequency and of about the same type in juvenile delinquents as in criminals. Endocrine treatment of the specific endocrine condition in juvenile delinquents has resulted in a correction of the delinquent behaviour. Juvenile delinquency and its sequel, crime, can be prevented by proper attention to the status of the different endocrines which contribute to the development of the normal social personality during childhood and adolescence.

C. S. R.

[170] Mental disorders in siblings.-Doncaster George Humm. Amer. Jour. Psychiat., 1932, xii, 239.

THIs study was undertaken in the hope of gaining a better understanding of the causation of persistent criminalism, manic-depressive psychosis, dementia præcox, epilepsy, and mental deficiency. A particular object was to determine the relative importance of hereditary and environmental factors in that causation. The material especially gathered for this study consisted of 858 siblings of patients suffering from these disorders. Access was also secured to 214 pairs of twins, one or both of each pair being affected by one of these disorders. Of these 138 were dizygotic and 76 were classified as probably monozygotic. 